\title{
Alleviation of Salt Stress by Enterobacter sp. EJ01 in Tomato and Arabidopsis Is Accompanied by Up-Regulation of Conserved Salinity Responsive Factors in Plants
}

\author{
Kangmin Kim ${ }^{1}$, Ye-Jin Jang ${ }^{1}$, Sang-Myeong Lee ${ }^{1,2}$, Byung-Taek $\mathrm{Oh}^{1,2}$, Jong-Chan Chae ${ }^{1,2}$, and \\ Kui-Jae Lee ${ }^{1,2, *}$
}

\begin{abstract}
Microbiota in the niches of the rhizosphere zones can affect plant growth and responses to environmental stress conditions via mutualistic interactions with host plants. Specifically, some beneficial bacteria, collectively referred to as Plant Growth Promoting Rhizobacteria (PGPRs), increase plant biomass and innate immunity potential. Here, we report that Enterobacter sp. EJ01, a bacterium isolated from sea china pink (Dianthus japonicus thunb) in reclaimed land of Gyehwa-do in Korea, improved the vegetative growth and alleviated salt stress in tomato and Arabidopsis. EJ01 was capable of producing 1-aminocy-clopropane-1-carboxylate (ACC) deaminase and also exhibited indole-3-acetic acid (IAA) production. The isolate EJ01 conferred increases in fresh weight, dry weight, and plant height of tomato and Arabidopsis under both normal and high salinity conditions. At the molecular level, short-term treatment with EJ01 increased the expression of salt stress responsive genes such as DREB2b, $R D 29 A, R D 29 B$, and $R A B 18$ in Arabidopsis. The expression of proline biosynthetic genes (i.e. P5CS1 and P5CS2) and of genes related to priming processes (i.e. MPK3 and MPK6) were also up-regulated. In addition, reactive oxygen species scavenging activities were enhanced in tomatoes treated with EJ01 in stressed conditions. GFP-tagged EJ01 displayed colonization in the rhizosphere and endosphere in the roots of Arabidopsis. In conclusion, the newly isolated Enterobacter sp. EJ01 is a likely PGPR and alleviates salt stress in host plants through multiple mechanisms, including the rapid up-regulation of conserved plant salt stress responsive signaling pathways.
\end{abstract}

\footnotetext{
${ }^{1}$ Division of Biotechnology, College of Environmental and Bioresource Sciences, ${ }^{2}$ Advanced Institute of Environment and Bioscience, Chonbuk National University, Iksan 570-752, Korea

*Correspondence leekj@jbnu.ac.kr

Received 30 August, 2013; revised 16 December, 2013; accepted 18 December, 2013; published online 19 February, 2014
}

Keywords: Arabidopsis, Enterobacter, PGPR, ROS, salt stress

\section{INTRODUCTION}

Soil salinity adversely affects plant growth and has become one of the major limiting factors in crop productivity worldwide. Mechanistically, high salinity primarily disrupts the cellular osmotic balance by lowering the water potential inside cells in a similar fashion to stresses induced by drought and freezing (Krasensky and Jonak, 2012). Prolonged salt stresses additionally cause ion toxicity due to increased concentrations of $\mathrm{Na}^{+}$and $\mathrm{Cl}^{-}$ions. Such unfavorable conditions subsequently induce oxidative stresses by generating reactive oxygen species (ROS) such as superoxide, hydrogen peroxide, singlet oxygen, and hydroxyl radicals, which are detrimental to cell viability.

However, plants have adapted to salt stresses via conserved signal transduction pathways that activate various stress avoidance and tolerance mechanisms (Xiong et al., 2002). In these processes, a phytohormone Abscisic acid (ABA) is known to play major roles via modulating the expression of genes that possess ABA-responsive elements (ABREs) in the promoter regions. Another cis-acting element known as dehydrationresponsive element (DRE) also plays an important role in salt stress responses in plants. In the case of Arabidopsis, RD29A and $R D 29 B$ are representative regulons of $A B R E$ and DRE, and their transcription is modulated by upstream transcription factors, DRE-binding proteins (DREB)-like genes. As a result of such signaling, diverse functional components like Late Embryogenesis Abundant (LEA) genes (e.g., RAB18), osmolytes, antioxidants, and reactive oxygen species (ROS) scavengers are activated and mitigate the oxidative damage caused by salinity (Lata and Prasad, 2011; Xiong et al., 2002; Zhu, 2002).

Various bacteria collectively referred to as Plant Growth Promoting Rhizobacteria (PGPRs) colonize the rhizosphere of many plant species and have beneficial effects on host plant growth, which is manifested physiologically as increased plant growth and reduced susceptibility to diseases (Bulgarelli et al., 2013). Many PGPRs exhibit properties of phosphorus solubil zation and siderophore production, which allow host plants to efficiently uptake phosphorus- and iron-derived nutrients from the soil, respectively. In addition, PGPRs often interfere with the biosynthesis of phytohormones, especially auxin and ethylene. Auxins are majorly produced from indole-3-acetamide (IAM) 
and indole-3-pyruvate (IPyA), precursors excreted from many soil- and plant-associated bacteria (Dodd and Perez-Alfocea, 2012; Spaepen and Vanderleyden, 2011). The direct linkage between bacterial indole acetic acid (IAA) production and modified root architecture of the host was clearly demonstrated between $A$. brasilense and wheat (Spaepen et al., 2007). However, IAA biosynthesis per se did not account for the beneficial effects of PGPR, because IAA knockout mutants still promoted plant growth (Spaepen and Vanderleyden, 2013). On the other hand, the level of ethylene, a phytohormone involved in senescence and growth inhibition of plants (Bulgarelli et al., 2013), has also been considered as a major indicator of PGPR effects on host plants. Many microbes excrete 1-aminocyclopropane1-carboxylate deaminase (ACCD), which is encoded by the conserved acdS gene, and degrade the ethylene precursor ACC to ammonia and $\alpha$-ketobutyrate, thereby reducing the level of ethylene. In addition, volatile compounds (VOCs) such as 3hydroxy-2-butanone (acetoin) and 2,3-butanediol generated by microbes have also been suggested to modulate host growth (Ryu et al., 2003). As mentioned, the modes of action of PGPRrelated factors are thus diverse and complex, but the mechanistic basis is still largely unknown, especially at the molecular level.

Several PGPRs have also been reported to enhance the drought and salt stress resistance of their hosts (Dodd and Perez-Alfocea, 2012; Yang et al., 2009). For example, Achromobacter piechaudii ARV8 producing ACCD conferred tolerance to drought and salt stresses in peppers and tomato (Mayak et al., 2004a; 2004b). Arabidopsis treated with Bacillus subtilis GB03 also showed enhanced salt tolerance (Zhang et al., 2008). In recent reports, combinatorial treatments of multiple microbes boosted salt tolerance in mung bean, tomato, and brahmi (Ahmad et al., 2011; Bharti et al., 2013; Shen et al., 2012). At the mechanistic level, such tolerance is largely explained by means of water homeostatsis, osmolyte accumulation, antioxidants, hormone homeostasis, the production of ACCD and volatile compounds, and sodium uptake/transport (Dodd and Perez-Alfocea, 2012; Yang et al., 2009). Altered levels of the stress phytohormone ABA were mainly attributed to stress resistance (Aroca et al., 2006; Jahromi et al., 2008). PGPRs isolated from halophytic weeds produce $A B A$ in vitro and enhanced salt tolerance in soybean (Naz et al., 2009). The production of ACCD was also suggested to be linked to drought tolerance, because abiotic stresses concomitantly increased the level of ethylene by up-regulating biosynthetic genes (Achard et al., 2006; Cao et al., 2006). Regardless of reports suggesting the positive effects of PGPRs in the saline rhizosphere, however, the modes of microbial action and their phyto-effects are multifarious and have yet to be delineated in detail.

In this study, we aimed to isolate a bacterium beneficial to plant growth and salt stress resistance, and investigated the effects of a PGPR on the expressions of conserved stressresponsive genes. Enterobacter sp. EJ01 facilitated plant growth and increased the salt tolerance of Arabidopsis and tomato via colonization of the host root tissues. The mechanisms underlying the acquired salt tolerance were apparently complex and likely included multiple physiological processes. Bacterial treatment rapidly induced the post-transcriptional modulation of salt stress responses of the hosts. Our data suggest that EJ01driven salt tolerance includes the rapid activation of conserved salt stress-responsive signaling pathways in host plants.

\section{MATERIALS AND METHODS}

Plant materials and growth conditions

Arabidopsis thaliana ecotype Columbia (Col-0) and tomato (Lycopersicon esculentum var. Mill) were used in this study. Seeds were surface sterilized with $2 \%$ sodium hypochlorite for $5 \mathrm{~min}$, followed by rinsing with sterile water. For germination of Arabidopsis, seeds were put on media containing half-strength MS media (Duchefa, Japan) and $0.5 \%$ sucrose under fluorescent light ( $15 \mathrm{~h}$ light $/ 9 \mathrm{~h}$ dark) at $22^{\circ} \mathrm{C}$. Then, ten-day-old Arabidopsis seedlings were transferred to plastic pots $(5 \times 5 \times 5$ $\mathrm{cm}$ ) filled with perlite and vermiculite (1:1) and grown further in soil under the same light regime. For tomato, seeds were germinated in wet filter paper for five days. Seedlings were then transferred to plastic pots $(7 \times 7 \times 7 \mathrm{~cm})$ filled with horticultural soil mix (cocopeat $60 \%$, peat moss $20 \%$, vermiculite $10 \%$, perlite $7 \%$, seaweed mix $3 \%$ ) and grown under the same conditions described for Arabidopsis.

To monitor plant growth and salt tolerance, two days after transfer to pots, Arabidopsis and tomato seedlings were inoculated with bacterial suspensions prepared as follows. Enterobacter $\mathrm{sp}$. EJ01, the bacterium isolated in the current study, was cultured in LB broth overnight at $30^{\circ} \mathrm{C}$, and cell pellets were harvested and resuspended with sterile water to achieve an $\mathrm{OD}_{600}$ of 0.5 . Ten $\mathrm{ml}$ of this suspension was then added to the soil around plant roots. Arabidopsis were watered by irrigation with 1× Hoagland solution (Hoagland and Arnon, 1950) with and without $200 \mathrm{mM} \mathrm{NaCl}$ every third day. Arabidopsis pots were filled with vermiculite and perlite containing no nutrients. In the case of tomato, the pots were filled with nutrientcontaining horticultural soil and were irrigated with distilled water with or without $200 \mathrm{mM} \mathrm{NaCl}$ every three days. After one month, the plant height, fresh weight, and dry weight were measured.

Isolation and taxonomical identification of bacteria

One gram of rhizosphere soil including root tissues of sea china pink (Dianthus japonicus thunb), a halophyte plant species, in reclaimed land of Gyehwa-do, Korea were ground in a mortar and inoculated in a flask containing $50 \mathrm{ml}$ of Dworkin-Foster (DF) salt minimal medium (Dworkin and Foster, 1958) mixed with $0.2 \% \mathrm{ACC}(\mathrm{v} / \mathrm{v})$. The flask was incubated on a rotary shaker at $180 \mathrm{rpm}$ for $48 \mathrm{~h}$ at $25^{\circ} \mathrm{C}$. Subsequently, serial dilutions $\left(10^{-5}\right.$ to $10^{-6}$ ) were plated again on DF salt minimal agar medium containing $0.2 \%$ ACC. After being incubated at $25^{\circ} \mathrm{C}$ for 2 days, distinct colonies were selected and subjected to further characterization.

For taxonomical identification of the finally chosen bacteria, the total genomic DNA of bacteria was extracted using a DNA extraction kit (GeneAll, Korea). The 16S rRNA gene was amplified by PCR using universal primers, 27F (5'-AGAGTTTG ATCATGGCTCAG- ${ }^{\prime}$ ) and 1522R (5'-AAGGAGGTGATCCAG CCGAC- $\left.3^{\prime}\right)$. The amplified products were then subjected to gel extraction and submitted for nucleotide sequencing.

\section{Measurement of IAA production and ACC deaminase activity}

The production of IAA was determined according to the method of Gordon and Weber (1951). Bacteria were inoculated in DF minimal medium supplemented with $0.05 \%$ L-tryptophan as an IAA precursor. Samples were cultured at $30^{\circ} \mathrm{C}$ for $48 \mathrm{~h}$, and then cell cultures were centrifuged at $13,000 \times g$. The supernatant was filtrated and then mixed with $150 \mathrm{ml}$ of concentrated $\mathrm{H}_{2} \mathrm{SO}_{4}, 250 \mathrm{ml}$ of distilled water, and $7.5 \mathrm{ml}$ of $0.5 \mathrm{M} \mathrm{FeCl}_{3}$. 
$6 \mathrm{H}_{2} \mathrm{O}$. After 30 min of incubation at room temperature, the absorbance at $535 \mathrm{~nm}$ was measured, and the concentration was determined based on a standard curve drawn with a series dilution of IAA (Sigma-Aldrich, USA).

The activity of ACC deaminase was also colorimetrically estimated by measuring the amount of $\alpha$-ketobutyrate produced from the cleavage of ACC (Penrose and Glick, 2003). Bacterium was cultured in LB broth overnight at $30^{\circ} \mathrm{C}$. The cell pellet was retrieved and transferred to DF salt minimal medium containing $3 \mathrm{mM} \mathrm{ACC}$ as the sole nitrogen source. When growth reached the late log phase (i.e. $\mathrm{OD}_{600}=1.0$ ), bacterial cell pellets were harvested, followed by two washes with $0.1 \mathrm{M}$ Tris$\mathrm{HCl}, \mathrm{pH} 7.6$ and $\mathrm{pH}$ 8.5, respectively. Toluene was added to the cell suspension and then immediately assayed for ACCdeaminase activities. To create a standard curve, $200 \mu \mathrm{l}$ of $\alpha$ ketobutyrate solutions at known concentrations were mixed with $300 \mu \mathrm{l}$ of $2 \%$ 2,4-dinitrophenylhydrazine dissolved in $2 \mathrm{M}$ $\mathrm{HCl}$. The mixture was vortexed and incubated at $30^{\circ} \mathrm{C}$ for 30 min. Phenylhydrazone color was developed by the addition of $2.0 \mathrm{ml}$ of $2 \mathrm{M} \mathrm{NaOH}$ and then measured for absorbance at 540 $\mathrm{nm}$.

\section{Bacterial colonization}

Bacterial strains expressing GFP were generated by fourparental mating conjugation. The recipient strain (i.e. Enterobacter sp. EJ01), E. coli XL1-Blue containing the plasmid pBK-

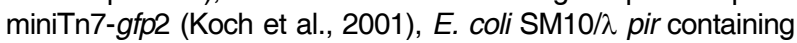
the plasmid pTNS3, and the helper strain E. coli HB101 containing the plasmid pRK2013 were grown separately overnight in LB broth in the presence of relevant antibiotics at $37^{\circ} \mathrm{C}$ with shaking. $100 \mu \mathrm{l}$ of each cell culture was taken and washed twice with LB pre-warmed to $37^{\circ} \mathrm{C}$. The cultures were combined and spotted on pre-warmed solid LB and incubated at $37^{\circ} \mathrm{C}$ for $6 \mathrm{~h}$. Cells were suspended using saline solution $(0.85 \%$ $\mathrm{NaCl})$ and plated on LB-agar supplemented with gentamycin (200 $\mu \mathrm{g} / \mathrm{ml}$ : selection for pBK-miniTn7-gfp2 insertion) and ampicillin $\left(30 \mu \mathrm{g} / \mathrm{ml}\right.$ : for EJ01) and incubated overnight at $37^{\circ} \mathrm{C}$ (Choi and Schweizer, 2006). Final colonies were selected, and their 16S rRNA genes were amplified and sequenced for confirmation. Selected bacterial strains were grown overnight in LB media, and cell pellets were resuspended to achieve $\mathrm{OD}_{600}=$ 0.5 with deionized water. Cell suspensions were administered to Arabidopsis seedlings grown vertically in a plate. The entirety of the roots was examined for GFP fluorescence using an AX10 fluorescent microscope (Zeiss, Sweden) or LSM 510 META confocal laser scanning microscope (Zeiss, Sweden).

\section{Quantitative measurement of expression of stress responsive genes}

Total RNA was isolated from whole seedlings treated with bacterial suspension $\left(\mathrm{OD}_{600}=0.5\right)$ for six $\mathrm{h}$, using TRIzol reagent (Invitrogen, USA). The first strand of cDNA was synthesized using one microgram of total RNA as a template and oligo dT primers (Takara, Japan). Diluted cDNAs were used as templates for real-time qRT-PCR using SYBR ${ }^{\circledR}$ Premix Ex Taq ${ }^{\mathrm{T}}$ (Takara, Japan) with a Rotor-Gene 6000 real-time rotary analyzer (Corbett Life Science, Australia). The expression levels of target genes were presented as a ratio relative to the expression of the reference gene ACTIN2.

Measurement of ROS scavenging activity

ROS scavenging activities were assessed by measuring DPPH and APX activities. The neutralization of DPPH, a stable radical, was determined by a modification of a previously described protocol (Blois, 1958). A reaction mixture containing $1.5 \mathrm{ml}$ of $0.2 \mathrm{mM}$ DPPH dissolved in $80 \%$ ethanol and $300 \mu \mathrm{l}$ of plant extract was incubated at $25^{\circ} \mathrm{C}$ for $30 \mathrm{~min}$, and then the absorbance at $540 \mathrm{~nm}$ was measured using a Shimadzu spectrophotometer UV-1800 (Shimadzu, Japan). All measurements were performed in triplicate. DPPH radical scavenging activity (expressed as a percentage) was calculated as [( $\left.\mathrm{Abs}_{\mathrm{DPPH}}-\mathrm{Abs}_{\text {sample }}\right) /$ $\left.\mathrm{Abs}_{\mathrm{DPPH}}\right] \times 100$.

The activities of APX were determined as described (Nakano and Asada, 1981). $0.5 \mathrm{~g}$ of the aerial parts of tomato plants were ground into fine powder, and the total proteins were extracted with $750 \mu \mathrm{l}$ of $50 \mathrm{mM}$ potassium phosphate buffer at $\mathrm{pH}$ 7.5 and $10 \mathrm{mM}$ ascorbate in the presence of $20 \mathrm{mg}$ of PVPP (Polyvinylpolypyrrolidone). After vigorously vortexing for $10 \mathrm{~min}$, the mixture was centrifuged at $12,000 \mathrm{rpm}$ for $15 \mathrm{~min}$ at $4^{\circ} \mathrm{C}$, and the supernatant (protein extract) was taken. The enzymatic reaction was initiated by adding $1372 \mu$ of $50 \mathrm{mM} \mathrm{K}$-phosphate at $\mathrm{pH} 7.5,75 \mu \mathrm{l}$ of $10 \mathrm{mM}$ ascorbate, $3 \mu \mathrm{l}$ of $100 \mathrm{mM} \mathrm{H}_{2} \mathrm{O}_{2}$, and $50 \mu \mathrm{l}$ of protein extract. The oxidation of ascorbate to dehydroascorbate was monitored colorimetrically by measuring the absorbance at $290 \mathrm{~nm}$. The activity was calculated as follows: APX activity $(\mu \mathrm{mol} / \mathrm{g}$ fresh weight $/ \mathrm{min})=(\Delta 290 / \mathrm{min}) \times[$ Total enzyme extraction buffer volume / volume of enzyme solution in reaction mixture $\times$ (volume of reaction mixture) / 2.8 (molecular extinction coefficient) $\times[1 /$ weight of sample tissue $(\mathrm{g})]$.

\section{Statistical analyses}

The significance of difference between bacterially infected and uninfected conditions in the growth promotion and salt tolerance of Arabidopsis and tomato were analyzed by student's $t$ tests. The quantitative real-time PCR of stress-responsive genes and ROS scavenging activities were assessed by the standard deviation of mean values from triplicates.

\section{RESULTS}

Isolation of Enterobacter sp. EJ01

To discover bacteria that could enhance the growth and salt resistance of host plants, we isolated bacteria from the rhizosphere of a halophytic plant, sea china pink, and screened for ACC-utilizing capability, a well-documented PGPR trait (Bulgarelli et al., 2013). As a result, isolate 4-1 exhibited the best growth in medium containing ACC (Supplementary Fig. 1), implying high levels of ACCD (ACC deaminase) production. The enzymatic activity of ACCD from isolate 4-1 was measured to be $27.09 \mu$ mole $\alpha$-ketobutyrate/mg of proteins of cell pellet after 16 $\mathrm{h}$ of growth in the presence of ACC as the sole nitrogen source. We also examined whether isolate 4-1 could produce the auxin. Noticeably, when grown in broth culture, isolate 4-1 secreted and accumulated IAA in proportion to incubation time (48.3 mg of IAA/ml of broth after $24 \mathrm{~h}$ of incubation). On the other hand, siderophore activity, known to facilitate iron uptake in roots, was rarely detected in the growth medium of 4-1 (data not shown).

To taxonomically identify isolate 4-1, its $16 \mathrm{~S}$ rRNA gene was amplified and sequenced. BLASTN (http://blast.ncbi.nlm.nih. gov) revealed that isolate 4-1 shared $99 \%$ homology with Enterobacter sp. R4-422 (GenBank accession no.: JQ659709.1) and Enterobacter sp. R4-368 (GenBank accession no.: CP005991). Based on this, we designated isolate 4-1 as Enterobacter sp. EJ01.

Enterobacter sp. EJ01 promotes growth and increases salt resistance of tomato seedlings

To test whether Enterobacter sp. EJ01 exerted PGPR activities 
and modulated plant responses against abiotic stresses, we infected tomato seedlings with EJ01 and monitored the growth behavior in the presence or absence of $200 \mathrm{mM} \mathrm{NaCl}$. After growing for one month, tomato seedlings infected with EJ01 displayed increased biomass, as determined by measurement of the length, fresh weight, and dry weight of the aerial parts of the plants (Fig. 1A). Compared to uninfected seedlings, EJ01infected tomatoes exhibited $37 \%$ and $20 \%$ increases in plant height under normal and high salinity conditions, respectively (Fig. 1B). More dramatic differences were observed in the biomass accumulation. Under normal conditions, EJ01 infection conferred increases in the fresh weight $(49 \%)$ and dry weight $(50 \%)$ of uninfected tomatoes (Figs. $1 \mathrm{C}$ and $1 \mathrm{D})$. In saltstressed conditions driven by $200 \mathrm{mM} \mathrm{NaCl}$, EJ01-infected seedlings still showed over $20 \%$ increases in both fresh and dry weight measurement compared to uninfected seedlings. The combined data strongly suggest that EJ01 promoted growth and alleviated the damage driven by salt stress in tomato seedlings.

\section{Enterobacter sp. EJ01 confers facilitated growth and increased salt resistance to Arabidopsis seedlings}

To further confirm the effect of EJ01 on the growth and salt stress tolerance of host plants, we extended our physiological studies to Arabidopsis. After growing for one month, Arabidopsis seedlings infected with EJ01 displayed increased biomass, as assessed by fresh weight and dry weight (Fig. 2A). Under stress-free conditions, the fresh weights of Arabidopsis Col-0 seedlings in EJ01-uninfected and -infected conditions were $0.28 \mathrm{~g}$ and $0.38 \mathrm{~g}$, respectively, a $30 \%$ difference (Fig. 2B). This clearly suggested that EJ01 infection boosted the vegetative growth of Arabidopsis. In the presence of $200 \mathrm{mM} \mathrm{NaCl}$, EJ01infected Col-0 seedlings also revealed higher fresh weight $(0.14$ g) than uninfected seedlings $(0.07 \mathrm{~g})$, representing a $100 \%$ increase. Dry weight measurement also showed similar results to the fresh weight. Compared to uninfected conditions, EJ01infected Col-0 seedlings exhibited $42 \%$ and $72 \%$ increases in dry mass under non-stressed conditions and with $200 \mathrm{mM} \mathrm{NaCl}$, respectively (Fig. 2C). The combined results strongly suggest that EJ01 promotes growth and alleviates salt stress in Arabidopsis.

\section{Enterobacter sp. EJ01 up-regulates the expression of} stress-responsive genes in the early stages of infection To delineate the mutualistic interaction between PGPRs and their host plants at the molecular level, the expressions of marker genes up-regulated in ABA-dependent and/or independent pathways in Arabidopsis were examined after a short period of bacterial infection (Fig. 3). Six hours after exposure to salt, the expressions of DREB2b, RD29A, RD29B, and $R A B 18$ were increased in the EJ01-infected Arabidopsis seedlings compared to the uninfected. Interestingly, $D R E B 2 b, R D 29 A$, and $R A B 18$ in EJ01-infected seedlings were up-regulated, even in the absence of salt treatment. On the other hand, increases in RD29B transcript levels in EJ01-infected seedlings were dependent on salt treatment. We also examined the expression levels of MPK3 and MPK6, which were reported to regulate the 'priming' processes in biotic stress resistance (Beckers et al., 2009). Interestingly, EJ01 infection alone increased the expressions of MPK3 and MPK6. This up-regulation of MPK3 and MPK6 was marginal but statistically significant. However, after salt treatment, no difference was observed between EJ01-free and EJ01-infected conditions. Taken together, EJ01 may positively activate MAPK signaling at the transcriptional level but in
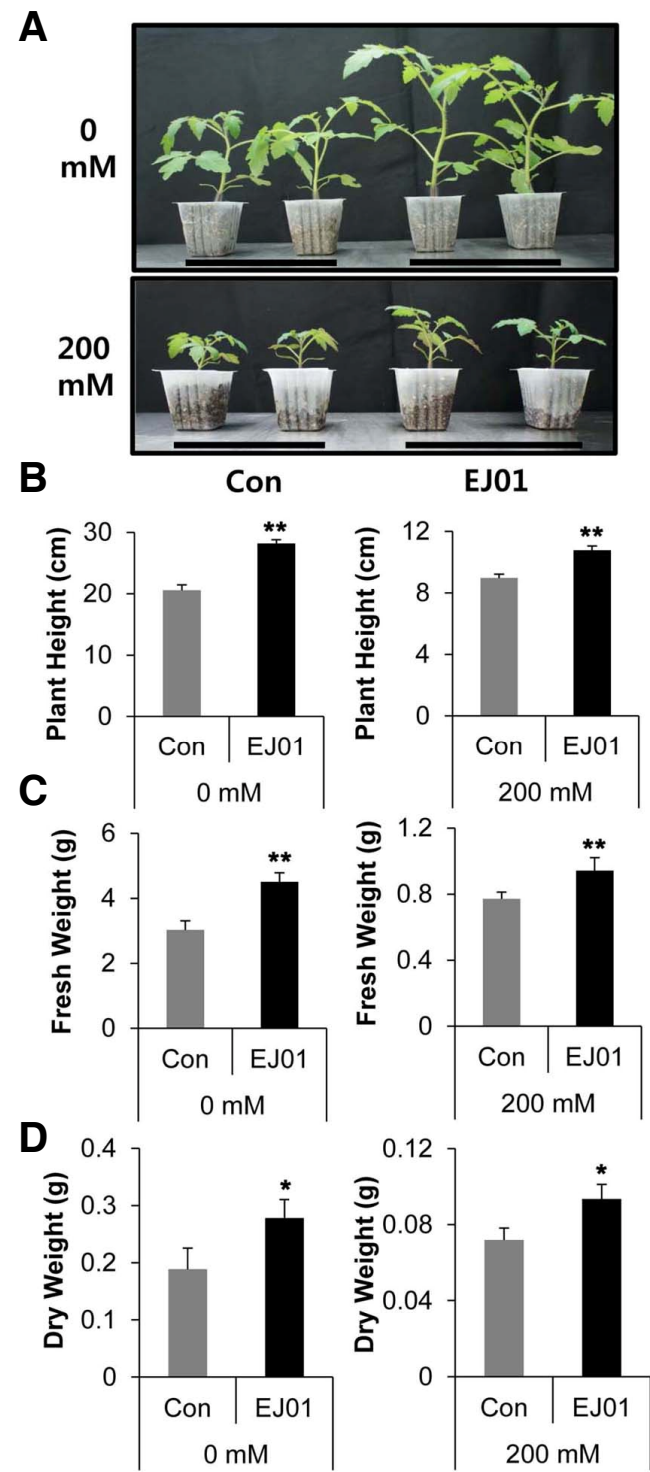

Fig. 1. The effects of Enterobacter sp. EJ01 on growth and salt resistance in tomato. (A) Representative tomato seedlings, uninfected or infected with EJ01, grown in the presence or absence of $200 \mathrm{mM} \mathrm{NaCl}$. (B) Average lengths of aerial parts of tomatoes under the same conditions. (C) Average fresh weight of tomato seedlings under the same conditions. (D) Average dry weight of tomato seedlings under the same conditions. Error bars indicate standard deviation $(n=16)$. ${ }^{*} P<0.05 ;{ }^{* *} P<0.01$ for Student's $t$-tests. After germination, tomato seedlings were transplanted to pots and treated with EJ01 suspensions near the root zone. Plants were irrigated with distilled water with or without $200 \mathrm{mM} \mathrm{NaCl}$ every three days. After one month, tomato seedlings were photographed, and then their aerial parts were removed and measured for fresh weight. Dry weight of aerial parts was measured after 2 days in a drying oven at $75^{\circ} \mathrm{C}$. Con: No bacterial infection; EJ01: EJ01-infected seedlings.

a limited scale.

The accumulation of compatible solutes such as sugars, glycine betaine, and proline is also a common stress avoidance mechanism in Arabidopsis. To investigate whether proline syn- 


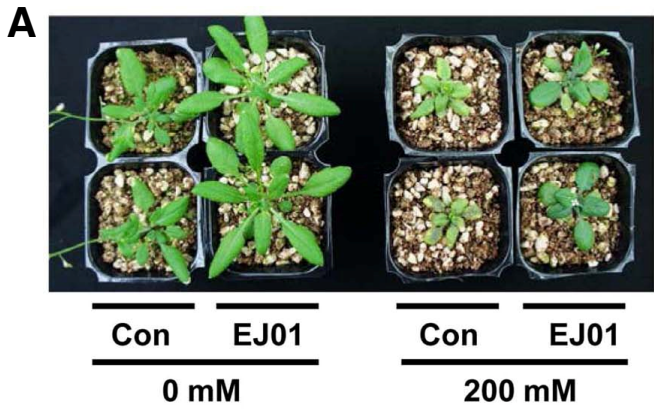

B

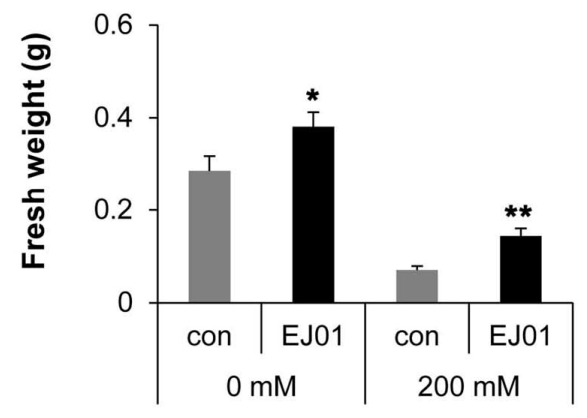

C

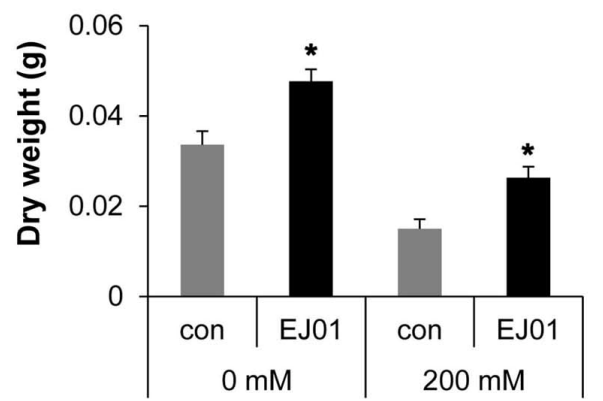

Fig. 2. The effect of Enterobacter sp. EJ01 on growth and salt tolerance of Arabidopsis. (A) Representative seedlings grown in pots treated or untreated with EJ01, under $0 \mathrm{mM}$ and $200 \mathrm{mM} \mathrm{NaCl}$. (B) Average fresh weight of individual Arabidopsis seedlings grown under the same conditions. (C) Average dry weight of individual Arabidopsis seedlings grown under the same conditions. Error bars indicate standard deviation $(n=25)$. ${ }^{*} P<0.05$; ${ }^{* *} P<0.01$ for Student's t-tests. Arabidopsis seedlings germinated on MS plates were transferred into pots and then treated with an EJ01 bacterial cell suspension or sterilized water. After two days, pots were watered by irrigation with $0.5 \times$ Hoagland nutrient solutions containing either $0 \mathrm{mM}$ or $200 \mathrm{mM} \mathrm{NaCl}$ every three days. Bacterial infection was repeated once more on the seventh day. After one month in pots, the Arabidopsis specimens were photographed. The aerial parts were then taken for measurement of the fresh weight. For dry weight determination, the seedlings taken for fresh weight measurement were dried at $70^{\circ} \mathrm{C}$ for two days and then weighed. Con, No bacterial treatment; EJ01, EJ01-treated seedlings.

thesis is facilitated by the EJ01 strain, we assessed the expression levels of P5CS1 and P5CS2 genes, which encode ratelimiting steps in proline biosynthesis. Notably, the expression of P5CS1 was highly up-regulated in EJ01-infected plants under stressed conditions. Interestingly, however, P5CS2 was also up-regulated by salt treatment but showed no difference be- tween bacteria-free and EJ01-infected conditions. On the other hand, the expression of $P R 1$, the most reliable read-out of the activation of defense, was also up-regulated, suggesting that EJ01 activated the innate immunity pathways as well as saltstress-responsive signaling.

ROS scavenging activities are increased in Arabidopsis seedlings exposed to Enterobacter sp. EJ01

Prolonged salt stress can cause oxidative stress and produce ROS, including superoxide, singlet oxygen, hydroxyl, and hydrogen peroxide inside cells. Such stressors can be countered by various antioxidants and enzymes directly related to stress resistance that detoxify ROS. To further explore the beneficial effects of EJ01 on the stress tolerance of host plants at a mechanistic level, the activities of common ROS scavenging systems in plants were quantitatively measured (Fig. 4). We first measured the activity of APX (ascorbate peroxidase), which is known to play key roles in detoxification under combinatorial stress (Koussevitzky et al., 2008) (Fig. 4A). EJ01-treated seedlings exhibited higher APX activities ( $3.2 \mu \mathrm{mol} / \mathrm{min} / \mathrm{g}$ fresh weight) under stressed conditions than did EJ01-free seedlings (2.6 $\mu \mathrm{mol} / \mathrm{min} / \mathrm{g}$ fresh weight). Similarly, DPPH (1,1-diphenyl-2-picrylhydrazyl), a widely used radical for antioxidant assays, was also neutralized more in EJ01-treated seedlings (55\%) than in EJ01free seedlings (46\%) (Fig. 4B). Taken together, EJ01-treated plants activated their detoxification processes against oxidative conditions driven by salt stress more efficiently than did untreated plants.

Spatial distribution of Enterobacter sp. EJ01 in host plant Next, to determine whether EJ01 colonized the interior of host plants, we monitored Green Fluorescent Protein (GFP) signals in the root tissues of Arabidopsis infected with recombinant EJ01 strains expressing GFP, which were generated by fourparental mating conjugation (see "Materials and Methods"). When Arabidopsis was treated with wild-type EJ01, no fluorescent signal was detected throughout the entire root system (data not shown). In contrast, Arabidopsis infected with GFPexpressing EJ01 strains (Fig. 5) exhibited strong GFP signals in the various regions in the roots. After one day of infection, EJ01 strains were colonized dominantly in the rhizosphere (Fig. 5A). However, after two days of infection, EJ01 were observed in the apoplastic regions of the maturation zone (Figs. 5B and $5 \mathrm{C}$ ), the vasculatures (Figs. $5 \mathrm{D}$ and $5 \mathrm{E}$ ), or the cytosolic compartment (Figs. 5F and 5G) in the roots. In some cases, EJ01 was specifically crowded around cells and appeared to intrude into the host tissue (Supplementary Fig. 2). On the other hand, no GFP signal was detected in the shoots (data not shown). Overall, EJ01 was distributed in the host tissue with indefinite spatial preference, indicating that EJ01 was dominantly rhizobacteria but also exhibited endospheric properties.

\section{DISCUSSION}

Many bacteria and fungi in the rhizosphere, known as PGPRs, facilitate the growth of their host plants (Kloepper and Schroth, 1978). Interestingly, when host plants are pre-treated (i.e. 'primed') with some bacteria, the host basal innate immunity can be increased, along with the induction of rapid protective responses to genuine pathogenic attacks at a systemic level, a phenomenon referred to as induced systemic resistance (ISR) (Kloepper et al., 2004). In addition, some PGPRs also confer resistance to abiotic stresses like salinity (Yang et al., 2009). Most previous studies have explicitly focused on the physiologi- 


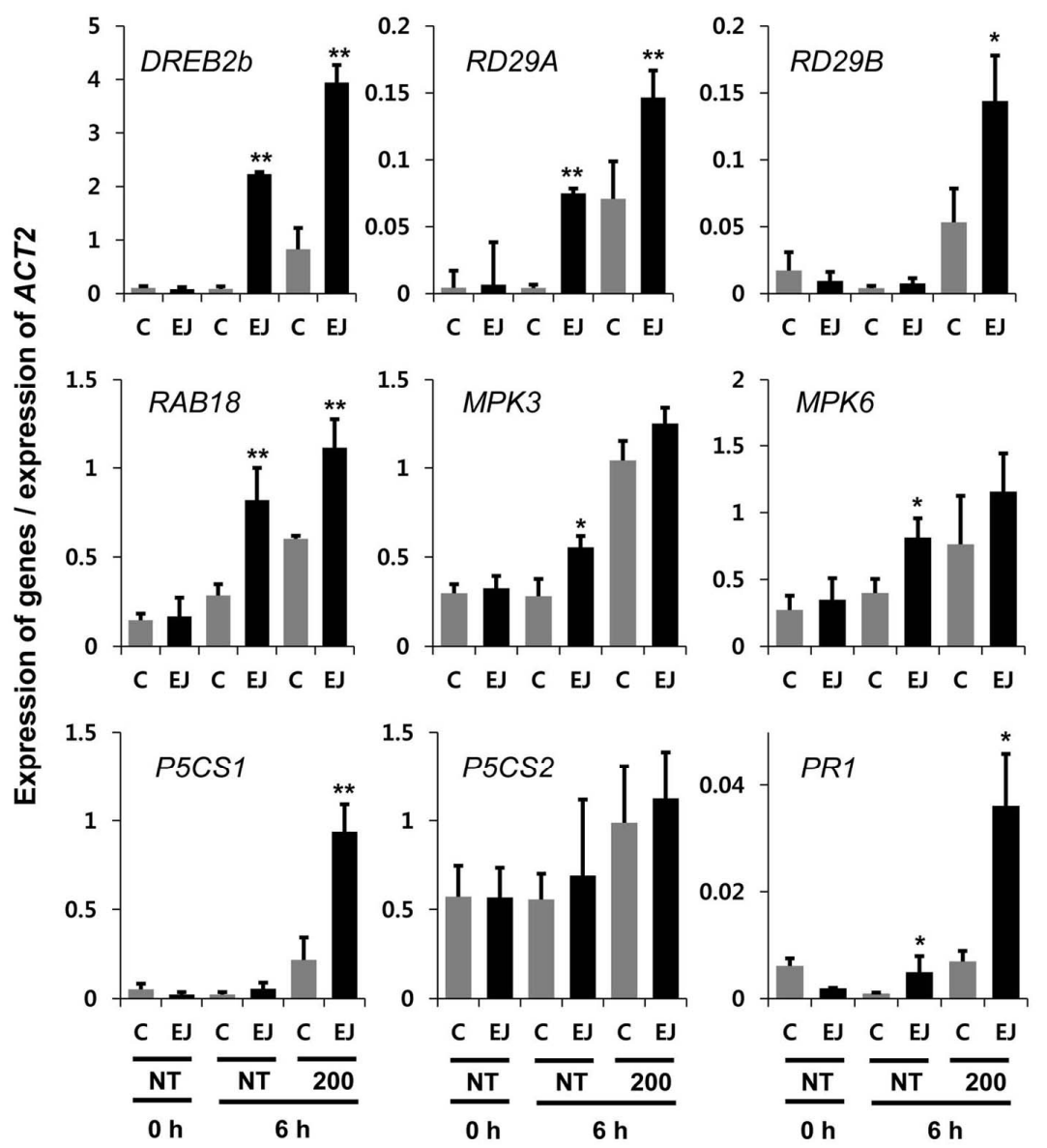

Fig. 3. Quantitative measurements of the expression levels of marker genes regulating stress responses in Arabidopsis. Tenday-old Arabidopsis seedlings were treated with Enterobacter sp. EJ01 under $0 \mathrm{mM}$ or $200 \mathrm{mM} \mathrm{NaCl}$ (see "Materials and $\mathrm{Me}$ thods"). After six $\mathrm{h}$, whole seedlings were collected and subjected to RNA isolation, followed by quantitative real-time RT-PCR. ACT2 was used as a control. Error bars indicate standard deviation $(\mathrm{n}=3)$. ${ }^{*} \mathrm{P}<$ $0.05 ;{ }^{* *} \mathrm{P}<0.01$ for Student's $t$-tests. $\mathrm{C}$, No bacterial treatment; EJ, EJ01-treated seedlings; NT, No addition of $\mathrm{NaCl}$; 200 , addition of $200 \mathrm{mM} \mathrm{NaCl}$. $0 \mathrm{~h}$, Zero time point of bacterial infection; $6 \mathrm{~h}$, six hours after bacterial infection.
A

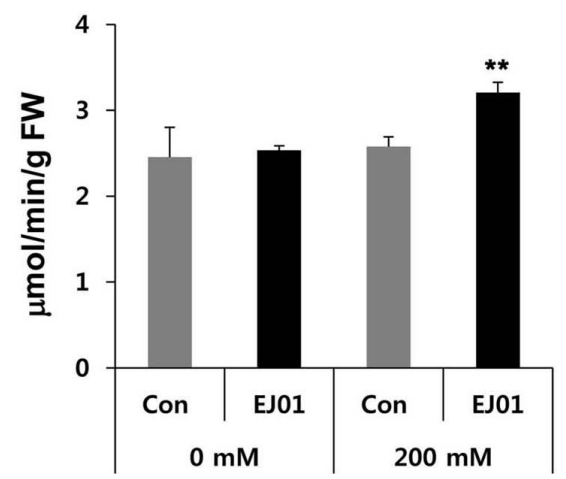

B

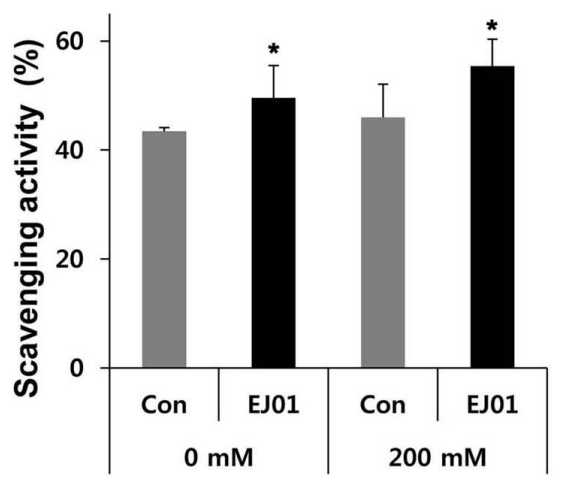

Fig. 4. The effect of strain EJ01 on ROS scavenging activities of tomatoes. (A) Activity of APX present in tomato seedlings. (B) Reduction of DPPH by tomato seedling extracts. Protein extracts from the aerial parts of onemonth-old tomatoes were used in APX and DPPH activity assays. For APX activity measurement, the reduction of hydrogen peroxide was monitored by the oxidation of ascorbate to dehydroascorbate. For DPPH, the reduction of DPPH was monitored colorimetrically, and scavenging activities were calculated as percentage decrease in

DPPH. Error bars indicate standard deviation $(n=3)$. ${ }^{*} \mathrm{P}<0.05$; ${ }^{* *} \mathrm{P}<0.01$ for Student's $t$-tests. Con, No bacterial infection; EJ01, EJ01infected tomato.

cal aspects of these effects, but the molecular basis thereof has not been investigated. Our study demonstrated that the newly isolated Enterobacter sp. EJ01 was capable of enhancing the growth and salt resistance of tomatoes and Arabidopsis. More importantly, stress tolerance driven by EJ01 is likely accompanied by bacteria-primed transcriptional activation of multiple stress-responsive factors prior to exposure to salt stresses, in a fashion similar to ISR against attacks by pathogens.

\section{The effects of EJ01 on phytohormone production}

EJ01 exhibits common PGPR characteristics such as ACCD and IAA production, suggesting that the growth promotion driven by EJ01 is likely ascribed to an increased level of auxin and the reduction of ethylene in the host plant. Combined genetic 

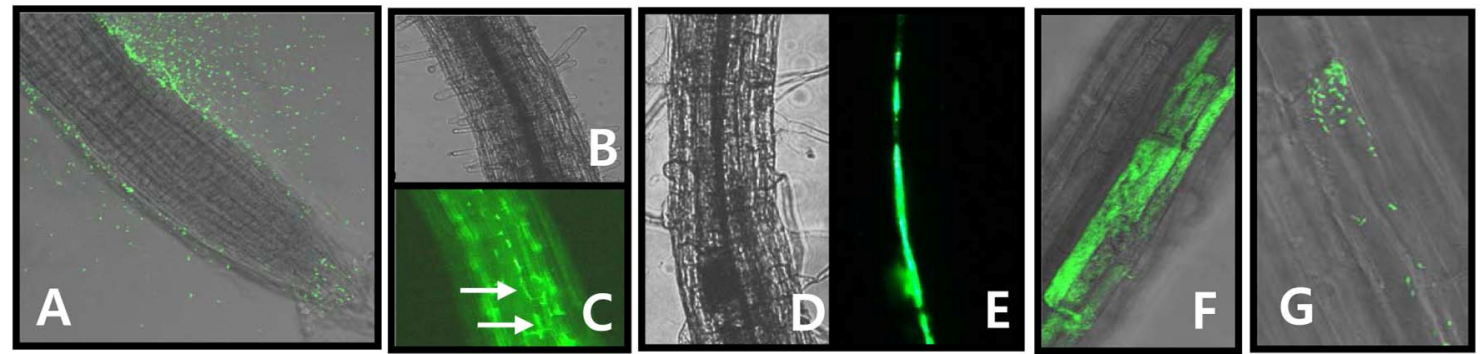

Fig. 5. Root colonization of recombinant Enterobacter sp. EJ01 expressing GFP. (A) The bacterial distribution in a longitudinal layer of rhizosphere zone. After one day of infection, meristemic zones of Arabidopsis root were monitored by optical sectioning. (B, C) The maturation zone of the roots. The arrows indicate the apoplastic regions or other cell periphery. $(D, E)$ The vasculature of the maturation zone of the roots. $(F)$ The cytosolic compartment of cell in elongation zone. (G) The enlarged image of one cell colonized by EJ01. (A, F, and G), observed by confocal fluorescent microscope; (B-E), observed by standard fluorescence microscope. GFP-containing EJ01 strains were generated by four parental mating (see "Materials and Methods") and applied to two-week-old Arabidopsis seedlings vertically grown in MS plate. After one and two days, GFP signals in the roots were monitored under fluorescent microscope or confocal laser scanning microscope. To prepare mating conjugation, the recipient strain EJ01, XL1-Blue E. coli containing the plasmid pBK-miniTn7-gfp2 (Koch et al., 2001), E. coli SM10/2 pir containing the plasmid pTNS3, and the helper strain E. coli HB101 containing the plasmid pRK2013 were used.

studies have demonstrated that the production of IAA or auxins from bacteria functionally correlates with increased growth of the host plants (Spaepen et al., 2007). For A. brasilense, a direct role for IAA production in the root architecture was demonstrated in wheat. In our study, EJ01 produced $43 \mathrm{mg} / \mathrm{ml}$ of IAA in liquid broth media, which was comparable with the amount produced by Klebsiella oxytoca Rs-5 $(42.14 \mathrm{mg} / \mathrm{ml}$ broth), a bacterial strain that increases salt stress tolerance in cotton (Liu et al., 2013). This could be interpreted that auxin produced by EJ01 might be responsible for growth promotion and salt tolerance. However, this must be regarded as uncertain, because auxin biosynthesis by several bacteria (e.g. Piriformospora indica) has been shown to be necessary only for root colonization (Hirano and Upper, 2000). In addition, Bacillus subtilis GB03 is known to facilitate the growth of host plants by inducing synthesis of auxin by its host, and not via IAA produced by itself (Zhang et al., 2007). Therefore, it is still unclear whether IAA produced by EJ01 is directly responsible for the observed growth promotion.

ACCD production has also been proposed as a key beneficial factor in PGPR action (Glick et al., 1998) and used as a selection criterion in primary screening for many PGPRs. In the current study, EJ01 was also isolated through screening for ACC utilization, and its ability to produce ACCD was confirmed. Previous reports demonstrated a correlation between ACCD production and the alleviation of salt stresses in host plants, but a direct correlation was not investigated on a wide scale (Mayak et al., 2004a; 2004b). Bacillus pumilus WP8 and Pseudomonas putida RBP1 fail to secrete ACCD, although they do increase salt tolerance (Shen et al., 2012). These findings imply that reduced ethylene production is not the sole factor accounting for salt tolerance in the strain EJ01.

\section{The roles of EJ01 in host stress adaptive signaling}

Under freezing and salt stresses, the activation of MKK2 (mitogen-activated kinase kinase 2) up-regulates the expression of stress-inducible marker genes and increases stress tolerance via MPK6 (Teige et al., 2004). The accumulation of mRNA of MPK3 and MPK6 was reported to be associated with ISR, which makes plants respond to (a)biotic stresses more rapidly and strongly (Beckers et al., 2009; Teige et al., 2004). Such actions are known to be primed by pre-exposure to moderate (a)biotic stimuli or stresses. This has been supported genetically by the impaired activation of MPK6 in $m k k 2$ null plants and severe stressed phenotypes (Teige et al., 2004). In our study, short-term treatment of Arabidopsis seedlings with EJ01 increased the expression levels of MPK3 and MPK6, even in the absence of $\mathrm{NaCl}$ (Fig. 3), which supported the hypothesis that pre-exposure to bacteria might induce pre-activation of stressresponsive pathways. Such molecular changes would expedite adaptive processes once genuine abiotic stresses are engaged However, considering the marginal increase in expression levels, the impact of such activation is largely speculative. Rather, the monitoring the status of post-translation modification would provide the better insights into physiological relevance of priming processes activated by EJ01.

The responses of plants to high salinity stresses are exemplified in the model plant, Arabidopsis (Zhu, 2002). The PGPR strain Paenibacillus polymyxa has been demonstrated to enhance the drought tolerance of Arabidopsis by stimulating the transcription of a drought-response gene, ERD15, and an ABAresponsive gene, $R A B 18$ (Timmusk and Wagner, 1999). These genes are known as late embryogenesis abundant (LEA) genes, whose expression is commonly up-regulated by cellular water deficits. $L E A$ genes have been proposed to be involved in the protection and repair of biomolecules inside the cell. Interestingly, $R A B 18$ was up-regulated even under PGPR treatments in the absence of salt stresses (Timmusk and Wagner, 1999). Currently, even though no precise mechanism is available, bacterial treatments to the roots could be perceived as a local dehydration-like signal which triggers salt-responsive pathways. Similar to these, in our study, EJ01 increased the expression of $L E A$ genes (e.g. $R A B 18$ and $R D 29 A$ ) and their transactivator (i.e. DREB2b), even in the absence of $\mathrm{NaCl}$ (Fig. 3). These results implicated that moderate exposure to biotic agents might activate signaling components located in the converged points governing multiple (a)biotic stress adaptation processes. On the other hand, the expression of RD29B, another LEA gene, was increased only when salt stresses were engaged. Differential expression patterns among $L E A$ genes might be related to ABA-dependency. Previously, the expression of $R D 29 A$ was known to be highly responsive to drought and cold stress- 
ses in both an ABA-dependent and ABA-independent fashion, whereas the promoter of $R D 29 B$ was regulated only by an ABA-dependent pathway (Msanne et al., 2011). In addition, $D R E B 2 b$, a transcription factor of both RD29 genes, displayed ABA-independent activation under osmotic stress conditions (Lata and Prasad, 2011; Zhu, 2002). Based on this, less induction of RD29B in EJ01-treated conditions (Fig. 3) implied that EJ01 activated salt-responsive pathways in ABA-independent fashion. Noticeably, this speculation was further supported by no increase in the expression levels of $A A O 3$, an ABA biosynthetic gene, when EJ01 was treated (Supplementary Fig. 3). In any event, this speculation must be experimentally examined, but the current results at least indicate that EJ01 activates early signaling events in stress-responsive pathways, providing benefits to host plants under salinity stress. Moreover, the expression of PR1 is also increased in EJ01- and $\mathrm{NaCl}$-treated seedlings (Fig. 3), suggesting that EJ01 treatment may also enhance innate immunity. Subsequently, EJ01 probably interferes with the upstream components of signaling-responsive pathways, which might impinge upon multiple pathways in (a)biotic stresses.

Turning to physiological aspects, some PGPRs produce antioxidants such as catalase and counter ROS-driven oxidative stresses. Bacillus safensis and Ochrobactrum pseudogregnonense can withstand osmotic stress via the elevation of antioxidant responses (Chakraborty et al., 2013). In our study, EJ01treated seedlings displayed slightly elevated ROS scavenging activities (Fig. 4), suggesting that the enhanced stress resistance conferred by EJ01 is at least partially due to cellular detoxification processes acting to counter oxidative stresses driven by high salinity. Proline accumulation is also a typical stress response induced by salt, and it is believed to function as an osmolyte or ROS scavenger. Even though proline biosynthesis was assayed only at the level of transcription, the expressions of P5CS1 and P5CS2 were greatly boosted in EJ01-exposed seedlings (Fig. 3). Proline accumulation levels have been shown to correlate highly with the transcript levels of P5CS genes, which regulate the rate-limiting step in proline biosynthesis (Yoshiba et al., 1997). However, the expression patterns of P5CS1 and P5CS2 were quite different from each other. Szekely et al. (2008) reported that the transcription of the P5CS1 was involved in abiotic stress adaptation. However, P5CS2 were more likely related to developmental processes such as embryo maturation. At any event, the up-regulation of P5CS genes indicates that EJ01 infection increases proline accumulation in Arabidopsis seedlings under high-saline cells.

Interestingly, our study clearly shows that EJ01 colonized the roots, and its modes of action must be endophytic (Fig. 5). However, considering the limited existence of EJ01 in the narrow zone of the rhizosphere and/or endosphere, it is intriguing how EJ01 exerted beneficial effects at the whole plant level due to the lack of a definite colonization pattern. Based on our results, EJ01 probably intrudes into plant tissue without spatial preference. In addition, the absence in the shoot tissues implicates that EJ01 might excrete elicitors to activate host signaling, which could be transmitted long distances to have a systemic impact at the whole plant level.

In conclusion, Enterobacter sp. EJ01 exerts beneficial roles in plant growth under unstressed and salt-stressed conditions. With respect to its molecular aspects, the mutually interactive modes of action in EJ01-facilitated growth and stress resistance in Arabidopsis and tomato include early responses activating osmolyte accumulation, ROS scavenging, and protein repair and protection driven by LEA genes. Monitoring the beneficial effects of EJ01 using appropriate mutant allelic plants of which stress signaling were modified would further elaborate its underlying mechanisms.

Note: Supplementary information is available on the Molecules and Cells website (www.molcells.org).

\section{ACKNOWLEDGMENTS}

This research was supported by the National Research Foundation of Korea (NRF) grant funded by the Korean government [Ministry of Education, Science and Technology (MEST)] (No. 2011-0020202) and the research funds of Chonbuk National University in 2013. The authors especially thank Gun-woong Lee, Kyeong-Taek Kim, and Eun-Ji Kim for technical assistance.

\section{REFERENCES}

Achard, P., Cheng, H., De Grauwe, L., Decat, J., Schoutteten, H., Moritz, T., Van Der Straeten, D., Peng, J., and Harberd, N.P (2006). Integration of plant responses to environmentally activated phytohormonal signals. Science 311, 91-94.

Ahmad, M., Zahir, Z.A., Asghar, H.N., and Asghar, M. (2011). Inducing salt tolerance in mung bean through coinoculation with rhizobia and plant-growth-promoting rhizobacteria containing 1aminocyclopropane-1-carboxylate deaminase. Can. J. Microbiol. 57, 578-589.

Aroca, R., Ferrante, A., Vernieri, P., and Chrispeels, M.J. (2006) Drought, abscisic acid and transpiration rate effects on the regulation of PIP aquaporin gene expression and abundance in Phaseolus vulgaris plants. Ann. Bot. 98, 1301-1310.

Beckers, G.J.M., Jaskiewicz, M., Liu, Y.D., Underwood, W.R., He, S.Y., Zhang, S.Q., and Conrath, U. (2009). Mitogen-activated protein kinases 3 and 6 are required for full priming of stress responses in Arabidopsis thaliana. Plant Cell 21, 944-953.

Bharti, N., Yadav, D., Barnawal, D., Maji, D., and Kalra, A. (2013). Exiguobacterium oxidotolerans, a halotolerant plant growth promoting rhizobacteria, improves yield and content of secondary metabolites in Bacopa monnieri (L.) Pennell under primary and secondary salt stress. World J. Microb. Biot. 29, 379-387.

Blois, M.S. (1958). Antioxidant determination by the use of a stable free radical. Nature 181, 1199-1200.

Bulgarelli, D., Schlaeppi, K., Spaepen, S., Ver Loren van Themaat, E., and Schulze-Lefert, P. (2013). Structure and functions of the bacterial microbiota of plants. Annu. Rev. Plant Biol. 64, 807-838.

Cao, W.H., Liu, J., Zhou, Q.Y., Cao, Y.R., Zheng, S.F., Du, B.X. Zhang, J.S., and Chen, S.Y. (2006). Expression of tobacco ethylene receptor NTHK1 alters plant responses to salt stress. Plant Cell Environ. 29, 1210-1219.

Chakraborty, U., Chakraborty, B.N., Chakraborty, A.P., and Dey, P.L. (2013). Water stress amelioration and plant growth promotion in wheat plants by osmotic stress tolerant bacteria. World J. Microbiol. Biotechnol. 29, 789-803.

Choi, K.H., and Schweizer, H.P. (2006). mini-Tn7 insertion in bacteria with secondary, non-glmS-linked attTn7 sites: example Proteus mirabilis HI4320. Nat. Protoc. 1, 170-178.

Dodd, I.C., and Perez-Alfocea, F. (2012). Microbial amelioration of crop salinity stress. J. Exp. Bot. 63, 3415-3428.

Dworkin, M., and Foster, J.W. (1958). Experiments with some microorganisms which utilize ethane and hydrogen. J. Bacteriol. 75, 592-603.

Glick, B.R., Penrose, D.M., and Li, J. (1998). A model for the lowering of plant ethylene concentrations by plant growth-promoting bacteria. J. Theor. Biol. 190, 63-68.

Gordon, S.A., and Weber, R.P. (1951). Colorimetric estimation of indoleacetic acid. Plant Physiol. 26, 192-195.

Hirano, S.S., and Upper, C.D. (2000). Bacteria in the leaf ecosystem with emphasis on Pseudomonas syringae - a pathogen, ice nucleus, and epiphyte. Microbiol. Mol. Biol. Rev. 64, 624-653.

Hoagland, D.R., and Arnon, D.I. (1950). The water-culture method for growing plants without soil. Vol. 347 revised. California Agriculture Experimental Station Center.

Jahromi, F., Aroca, R., Porcel, R., and Ruiz-Lozano, J.M. (2008). Influence of salinity on the In vitro development of Glomus intra- 
radices and on the In vivo physiological and molecular responses of mycorrhizal lettuce plants. Microb. Ecol. 55, 45-53.

Kloepper, J.W., and Schroth, M.N. (1978). Plant growth-promoting rhizobacteria on radishes. pp. 879-882 in: Proc. of the $4^{\text {th }}$ Internat. Conf. on Plant Pathogenic Bacter. Vol 2, Station de Pathologie Vegetale et Phytobacteriologie, INRA, Angers, France.

Kloepper, J.W., Ryu, C.M., and Zhang, S.A. (2004). Induced systemic resistance and promotion of plant growth by Bacillus spp. Phytopathology 94, 1259-1266

Koch, B., Jensen, L.E., and Nybroe, O. (2001). A panel of Tn7based vectors for insertion of the gfp marker gene or for delivery of cloned DNA into Gram-negative bacteria at a neutral chromosomal site. J. Microbiol. Meth. 45, 187-195.

Koussevitzky, S., Suzuki, N., Huntington, S., Armijo, L., Sha, W., Cortes, D., Shulaev, V., and Mittler, R. (2008). Ascorbate peroxidase 1 plays a key role in the response of Arabidopsis thaliana to stress combination. J. Biol. Chem. 283. 34197-34203.

Krasensky, J., and Jonak, C. (2012). Drought, salt, and temperature stress-induced metabolic rearrangements and regulatory networks. J. Exp. Bot. 63, 1593-1608.

Lata, C., and Prasad, M. (2011). Role of DREBs in regulation of abiotic stress responses in plants. J. Exp. Bot. 62, 4731-4748.

Liu, Y., Shi, Z.Q., Yao, L.X., Yue, H.T., Li, H., and Li, C. (2013). Effect of IAA produced by Klebsiella oxytoca Rs-5 on cotton growth under salt stress. J. Gen. Appl. Microbiol. 59, 59-65.

Mayak, S., Tirosh, T., and Glick, B.R. (2004a). Plant growth-promoting bacteria confer resistance in tomato plants to salt stress. Plant Physiol. Bioch. 42, 565-572.

Mayak, S., Tirosh, T., and Glick, B.R. (2004b). Plant growth-promoting bacteria that confer resistance to water stress in tomatoes and peppers. Plant Sci. 166, 525-530.

Msanne, J., Lin, J.S., Stone, J.M., and Awada, T. (2011). Characterization of abiotic stress-responsive Arabidopsis thaliana RD29A and $R D 29 B$ genes and evaluation of transgenes. Planta 234 , 97-107.

Nakano, Y., and Asada, K. (1981). Hydrogen-peroxide is scavenged by ascorbate-specific Peroxidase in spinach-chloroplasts. Plant Cell Physiol. 22, 867-880.

Naz, I., Bano, A., and Ul-Hassan, T. (2009). Isolation of phytohormones producing plant growth promoting rhizobacteria from weeds growing in Khewra salt range, Pakistan and their implication in providing salt tolerance to Glycine max L. Afr. J. Biotechnol. 8, 57-62.

Penrose, D.M., and Glick, B.R. (2003). Methods for isolating and characterizing ACC deaminase-containing plant growth-promoting rhizobacteria. Physiol. Plant 118, 10-15.

Ryu, C.M., Farag, M.A., Hu, C.H., Reddy, M.S., Wei, H.X., Pare, P. W., and Kloepper, J.W. (2003). Bacterial volatiles promote growth in Arabidopsis. Proc. Natl. Acad. Sci. USA 100, 4927-4932.

Shen, M., Kang, Y.J., Wang, H.L., Zhang, X.S., and Zhao, Q.X (2012). Effect of plant growth-promoting rhizobacteria (PGPRs) on plant growth, yield, and quality of tomato (Lycopersicon esculentum Mill.) under simulated seawater irrigation. J. Gen. Appl. Microbiol. 58, 253-262.

Spaepen, S., and Vanderleyden, J. (2011). Auxin and plantmicrobe Interactions. Cold Spring Harb. Perspect. Biol. 3, 1-13.

Spaepen, S., Vanderleyden, J., and Remans, R. (2007). Indole-3acetic acid in microbial and microorganism-plant signaling. Fems. Microbiol. Rev. 31, 425-448.

Teige, M., Scheikl, E., Eulgem, T., Doczi, F., Ichimura, K., Shinozaki, K., Dangl, J.L., and Hirt, H. (2004). The MKK2 pathway mediates cold and salt stress signaling in Arabidopsis. Mol. Cell 15, 141-152.

Timmusk, S., and Wagner, E.G.H. (1999). The plant-growth-promoting rhizobacterium Paenibacillus polymyxa induces changes in Arabidopsis thaliana gene expression: a possible connection between biotic and abiotic stress responses. Mol. Plant Microbe Interact. 12, 951-959.

Xiong, L.M., Schumaker, K.S., and Zhu, J.K. (2002). Cell signaling during cold, drought, and salt stress. Plant Cell 14, S165-S183.

Yang, J., Kloepper, J.W., and Ryu, C.M. (2009). Rhizosphere bacteria help plants tolerate abiotic stress. Trends Plant Sci. 14, 1-4.

Yoshiba, Y., Kiyosue, T., Nakashima, K., Yamaguchi-Shinozaki, K., and Shinozaki, K. (1997). Regulation of levels of proline as an osmolyte in plants under water stress. Plant Cell Physiol. 38 1095-1102.

Zhang, H., Kim, M.S., Krishnamachari, V., Payton, P., Sun, Y., Grimson, M., Farag, M.A., Ryu, C.M., Allen, R., Melo, I.S., et al. (2007). Rhizobacterial volatile emissions regulate auxin homeostasis and cell expansion in Arabidopsis. Planta 226, 839851.

Zhang, H., Kim, M.S., Sun, Y., Dowd, S.E., Shi, H.Z., and Pare, P. W. (2008). Soil bacteria confer plant salt tolerance by tissuespecific regulation of the sodium transporter HKT1. Mol. Plant Microbe Interact. 21, 737-744.

Zhu, J.K. (2002). Salt and drought stress signal transduction in plants. Annu. Rev. Plant Biol. 53, 247-273. 